Publ. Mat. 63 (2019), 343-360

DOI: 10.5565 /PUBLMAT6311912

\title{
LATTICE POINTS IN ELLIPTIC PARABOLOIDS
}

\author{
Fernando Chamizo and Carlos Pastor
}

\begin{abstract}
We consider the lattice point problem corresponding to a family of elliptic paraboloids in $\mathbb{R}^{d}$ with $d \geq 3$ and we prove the expected to be optimal exponent, improving previous results. This is especially noticeable for $d=3$ because the optimal exponent is conjectural even for the sphere. We also treat some aspects of the case $d=$ 2 , getting for a simple parabolic region an $\Omega$-result that is unknown for the classical circle and divisor problems.
\end{abstract}

2010 Mathematics Subject Classification: 11P21, 11L07.

Key words: Elliptic paraboloids, lattice point problem, exponential sums.

\section{Introduction}

Given a fixed compact subset $\mathcal{K}$ of $\mathbb{R}^{d}$ satisfying certain regularity conditions, the fundamental problem in lattice point theory consists in estimating

$$
\mathcal{N}(R)=\#\left\{\vec{n} \in \mathbb{Z}^{d}: R^{-1} \vec{n} \in \mathcal{K}\right\}
$$

for large values of $R$. The natural approximation is $|\mathcal{K}| R^{d}$, where $|\mathcal{K}|$ stands for the volume of $\mathcal{K}$, and one is interested in the optimal exponent

$$
\alpha_{\mathcal{K}}=\inf \left\{\alpha>0: \mathcal{N}(R)=|\mathcal{K}| R^{d}+O\left(R^{\alpha}\right)\right\} .
$$

For instance, when $\mathcal{K}$ is a convex body with smooth boundary and nonvanishing Gaussian curvature it is known $\alpha_{\mathcal{K}} \leq 131 / 208$ for $d=2$, and $\alpha_{\mathcal{K}} \leq d-2+r(d)$ with $r(d)=73 / 158$ for $d=3$ and $r(d)=\left(d^{2}+3 d+\right.$ 8) $/\left(d^{3}+d^{2}+5 d+4\right)$ for $d \geq 4[\mathbf{1 8}, \mathbf{1 2}]$.

Upper bounds for $\alpha_{\mathcal{K}}$ have been extensively studied for a great number of families of sets $\mathcal{K}$, but sharp results are scarce. Paradigmatic to the theory are the celebrated problems of Gauss and Dirichlet, dealing with the circle and a hyperbolic region of the plane (with extra conditions in the latter case to ensure compactness). The best known upper bound for both of them has recently been improved by Bourgain and Watt in [5] from Huxley's aforementioned result to $\alpha_{\mathcal{K}} \leq 517 / 824$ (conjecturally

The first author is partially supported by the MINECO grant MTM2014-56350-P. The second author has been supported by the "la Caixa"-Severo Ochoa international PhD programme at the Instituto de Ciencias Matemáticas (CSIC-UAM-UC3M-UCM). 
also valid for ellipses), still far from Hardy's conjecture $\alpha_{\mathcal{K}}=1 / 2$. The situation is not much better understood in three dimensions, where the best known upper bound for the sphere and the average of the class number (corresponding to a family of hyperboloids) is $\alpha_{\mathcal{K}} \leq 21 / 16$ [16, 7 , result extended to rational ellipsoids in [6]. The expected value of $\alpha_{\mathcal{K}}$ in these cases, and in general for $d \geq 3$, is $d-2$.

As the dimension increases the lattice point problem for the ball and rational ellipsoids becomes simpler, due to the higher regularity of the number of representations of integers by rational quadratic forms. This leads in a fairly easy manner to the sharp result $\alpha_{\mathcal{K}}=d-2$ for $d \geq 4$, contained in classical works. Less is known in the irrational case, where the inequality $\alpha_{\mathcal{K}} \leq d-2$ was finally achieved in [2] for $d \geq 9$ and later in [11] for $d \geq 5$. Surprisingly, the error term is, in contrast with the rational case, $o\left(R^{d-2}\right)$, and this led to a proof of the Davenport-Lewis conjecture about the gaps of the image of $\mathbb{Z}^{d}$ under irrational quadratic forms $[\mathbf{3}]$. For further reference on these and other lattice point problems related to number theory questions we refer the reader to $[\mathbf{1 9}]$.

It is surprising the little attention attracted by the remaining conic, the parabola, and its higher-dimensional analogues. The bidimensional case was addressed by Popov [26], proving the upper bound $\alpha_{\mathcal{K}} \leq 1 / 2$ for a variant of the lattice point problem $\mathcal{K}=\left\{|y| \leq c-(x+\beta)^{2}\right\}, c>0$, and the sharp result $\alpha_{\mathcal{K}}=1 / 2$ when $\beta=0$ and $c \in \mathbb{Q}$. This remarkable difference with the Gauss and Dirichlet problems is a consequence of the fact that the error term for the parabola can be expressed in terms of 1-dimensional quadratic exponential sums, and these can be finely estimated with simple Diophantine considerations. In fact in some particular cases this leads, via the evaluation of Gauss sums, to an explicit formula for the error term involving $L$-functions that seems to have been overlooked in the literature. We derive this formula for the case $c=1, \beta=0$ and show that it can be used to obtain a one-sided $\Omega$-result beyond what is known for both the circle and the hyperbola $[\mathbf{1 3}, \mathbf{2 8}]$, namely (see Proposition 5.2)

$$
\mathcal{N}(R)-|\mathcal{K}| R^{2}=\Omega_{-}\left(R^{1 / 2} \exp (c \sqrt{\log R} / \log \log R)\right) \quad \text { for any } c<\sqrt{2} \text {. }
$$

In higher dimensions the natural set to study is that of elliptic paraboloids of the form

$$
\mathcal{P}=\left\{(\vec{x}, y) \in \mathbb{R}^{d-1} \times \mathbb{R}:|y| \leq c-Q(\vec{x}+\vec{\beta})\right\}
$$

where $Q$ is a positive definite quadratic form, $\vec{\beta}$ is a fixed vector in $\mathbb{R}^{d-1}$, and $c$ a positive constant. The particular case $\vec{\beta}=0$ was considered in a slightly different form by Krätzel $[\mathbf{2 2}, \mathbf{2 3}]$, but his method only yielded the inequality $\alpha_{\mathcal{P}} \leq d-2$ under the strong assumptions $d \geq 5$ and 
$Q$ either rational or diagonal (proving, in the rational case, $\alpha_{\mathcal{P}}=d-2$ for $c \in \mathbb{Q}$ ). Partial results were given under weaker rationality assumptions in terms of the coefficient matrix $A=\left(a_{i j}\right)$ of $Q$. In particular, Krätzel obtained $\alpha_{\mathcal{P}} \leq d-5 / 3$ for $d \geq 3$ as long as $a_{12} / a_{11}, a_{22} / a_{11} \in \mathbb{Q}$. We improve these results:

Theorem 1.1. If $a_{12} / a_{11}, a_{22} / a_{11} \in \mathbb{Q}$ then the inequality $\alpha_{\mathcal{P}} \leq d-2$ holds for any $d \geq 3$. If moreover $\vec{\beta}=0, c \in \mathbb{Q}$, and $Q$ is rational then this is sharp.

Note that no assumptions are imposed on the remaining coefficients, and therefore this result extends the upper bound $\alpha_{\mathcal{P}} \leq d-2$ not only to $d=3,4$ and $\vec{\beta} \neq 0$, but also to a wider family of higher-dimensional paraboloids. The key step in the proof is the estimation of a certain quadratic exponential sum in two variables, which is done employing what can be considered a toy version of the circle method. Bounds this precise are out of reach for the exponential sums arising in most lattice point problems, and this accounts for the striking difference between our theorem and what is currently known for ellipsoids and hyperboloids. In fact, to the best of our knowledge, Theorem 1.1 constitutes the first sharp result for a lattice point problem in three dimensions.

The structure of the paper is as follows: First we revisit the twodimensional case, deriving the exact formula for the error term. After this we devote $\S 3$ to estimate the quadratic exponential sum involved in the proof of the first part of Theorem 1.1, and $\S 4$ to the proof itself. Finally in $\S 5$ we prove some $\Omega$-results that readily imply the second part of Theorem 1.1, together with more precise two-sided $\Omega$-results for the parabolic region considered in $\S 2$.

Along this paper, $e(x)$ is an abbreviation for $e^{2 \pi i x}$ and $\epsilon$ denotes an arbitrarily small positive quantity, that may change in value at each appearance. We employ $f(x)=O(g(x))$ and $f(x) \ll g(x)$ indistinctly to mean that $\limsup |f| /|g|<\infty$ and $f(x)=o(g(x))$ meaning $\lim f / g=0$. The negation of the latter is denoted by $f(x)=\Omega(g(x))$, while the symbol $\Omega_{+}$(or $\Omega_{-}$) is employed to specify that the positive (or negative) part of $f(x)$ is $\Omega(g(x))$.

\section{A parabolic region}

Popov gave in $[\mathbf{2 6}]$ an asymptotic for the number of lattice points under a parabola, i.e. in the region $\left\{0 \leq y \leq a x^{2} \leq c\right\}$ of the XY plane. His method readily applies to the region of the form $\left\{|y| \leq c-(x+\beta)^{2}\right\}$ mentioned in the introduction, yielding $\alpha_{\mathcal{K}} \leq 1 / 2$. In fact it is possible to give a very short proof of this result in few lines, essentially adapting 
the forthcoming argument of $\S 4$ to dimension two, and then appealing to classic estimates of quadratic sums [21, Theorem 8.1]. When the dilation is integral these quadratic sums can be evaluated explicitly, and the resulting formula relates the error term for this lattice point problem to the class number associated to a family of imaginary quadratic fields. We credit Professor Antonio Córdoba for pointing out the relation with the class number in the early 90's while he was the Ph.D. advisor of the first named author.

To illustrate the situation, we consider in this section a simple parabolic region

$$
\mathcal{P}_{2}=\left\{(x, y) \in \mathbb{R}^{2}:|y| \leq 1-x^{2}\right\},
$$

and denote by $\mathcal{N}_{2}(R)$ the number of lattice points in $\mathcal{P}_{2}$ scaled by $R$.

Theorem 2.1. Let $N$ be an odd positive integer and let $N^{*}$ be the greatest square dividing $N$. Then

$$
\mathcal{N}_{2}(N)=\left|\mathcal{P}_{2}\right| N^{2}+\frac{1}{3}+2 \sqrt{N^{*}}-\frac{4}{\pi} \sum_{\substack{d \mid N \\ d \equiv 3(4)}} \sqrt{d} L\left(1, \chi_{-d}\right),
$$

where $L\left(1, \chi_{-d}\right)$ is the $L$-function corresponding to the Kronecker symbol $\chi_{-d}=\left(\frac{-d}{\cdot}\right)$.

With some effort the result can be extended, with modifications, to cover the even case.

Two particular cases of Theorem 2.1 deserve special attention. They will be used in $\S 5$ to obtain one-sided $\Omega$-results for this lattice point problem.

Corollary 2.2. If the prime factors of $N$ are of the form $4 k+1$, then

$$
\mathcal{N}_{2}(N)-\left|\mathcal{P}_{2}\right| N^{2}=\frac{1}{3}+2 \sqrt{N^{*}} .
$$

Corollary 2.3. If $N$ is squarefree then

$$
\mathcal{N}_{2}(N)-\left|\mathcal{P}_{2}\right| N^{2}=\frac{7}{3}-4 \sum_{\substack{d \mid N \\ d \equiv 3(4)}} \omega_{d} h(-d)
$$

where $h(-d)$ is the class number of the integer ring of $\mathbb{Q}(\sqrt{-d})$ and $\omega_{d}=1$ except for $\omega_{3}=1 / 3$.

Proof: Apply Dirichlet class number formula in Theorem 2.1 for the fundamental discriminant $-d$. 
Proof of Theorem 2.1: Writing $\psi(x)=x-\lfloor x\rfloor-1 / 2$,

$$
\mathcal{N}_{2}(N)=2 \sum_{n=-N}^{N}\left(N-\frac{n^{2}}{N}\right)-2 \sum_{n=-N}^{N} \psi\left(-\frac{n^{2}}{N}\right) .
$$

The first sum is $\left(4 N^{2}-1\right) / 3$ and the area is clearly $\left|\mathcal{P}_{2}\right|=8 / 3$. Then

$$
\mathcal{N}_{2}(N)-\left|\mathcal{P}_{2}\right| N^{2}=-\frac{2}{3}-2 \sum_{n=-N}^{N} \psi\left(-\frac{n^{2}}{N}\right)=\frac{1}{3}-4 \sum_{n=1}^{N} \psi\left(-\frac{n^{2}}{N}\right)
$$

We now substitute

$$
\psi(x)=\Im \sum_{m=1}^{\infty} \frac{e(-m x)}{\pi m}+ \begin{cases}0 & \text { if } x \notin \mathbb{Z} \\ -1 / 2 & \text { if } x \in \mathbb{Z}\end{cases}
$$

Note that $N$ divides $n^{2}$ exactly $\sqrt{N^{*}}$ times in the range $1 \leq n \leq N$, and hence

$$
\mathcal{N}_{2}(N)-\left|\mathcal{P}_{2}\right| N^{2}=\frac{1}{3}+2 \sqrt{N^{*}}-\frac{4}{\pi} \sum_{m=1}^{\infty} \frac{1}{m} \Im G(m ; N),
$$

where $G(m ; N)$ is the quadratic Gauss sum $\sum_{n=1}^{N} e\left(m n^{2} / N\right)$. Let $d_{m}=$ $N / \operatorname{gcd}(m, N)$, the evaluation of $\Im G(m ; N)$ reads $[\mathbf{2 1}$, Exercise 4 of Chapter 3]

$$
\Im G(m ; N)=\left\{\begin{array}{lll}
0 & \text { if } d_{m} \equiv 1 & (\bmod 4), \\
\frac{N}{\sqrt{d_{m}}}\left(\frac{m d_{m} / N}{d_{m}}\right) & \text { if } d_{m} \equiv 3 & (\bmod 4) .
\end{array}\right.
$$

Substituting in (2.1) and noting that when $d_{m}$ is fixed $m d_{m} / N$ runs over all positive integers coprime to $d_{m}$, we have

$$
\mathcal{N}_{2}(N)-\left|\mathcal{P}_{2}\right| N^{2}=\frac{1}{3}+2 \sqrt{N^{*}}-\frac{4}{\pi} \sum_{\substack{d \mid N \\ d \equiv 3(4)}} \sqrt{d} \sum_{m=1}^{\infty} \frac{1}{m}\left(\frac{m}{d}\right) .
$$

By the quadratic reciprocity law for the Jacobi-Kronecker symbol $[\mathbf{2 1}$, $\S 3.5]$, the innermost sum equals $L\left(1, \chi_{-d}\right)$.

\section{Elliptical summation}

Consider the well known Hardy-Littlewood bound:

$$
\sum_{n=-N}^{N} e\left(n^{2} x\right) \ll q^{-1 / 2} N \quad \text { if }\left|x-\frac{a}{q}\right| \leq \frac{1}{4 q N} \text { with } q \leq 4 N
$$

and coprime to $a$. 
Although this result is implicit in the work [14], the reader looking for a closer statement may consult $[\mathbf{9}]$. Squaring the last inequality we have

$$
\sum_{(n, m) \in \mathcal{C}} e\left(\left(n^{2}+m^{2}\right) x\right) \ll q^{-1} N^{2} \quad \text { with } \mathcal{C}=[-N, N] \times[-N, N] .
$$

In principle it is not clear whether the square $\mathcal{C}$ can be replaced by a circle or ellipse. This is forced in our approach (with extra linear terms) to keep the symmetry. Proposition 3.1 below shows that this is possible losing at most a power of logarithm. The problem was addressed in [22] and $[\mathbf{2 3}]$ via the estimation of 2-dimensional exponential sums getting a weaker result, and recently in $[\mathbf{1 7}]$ when the sum has a certain smooth cut-off. Here we employ a simplified version of the circle method, taking advantage of the fact that only upper bounds are required on any arc.

For convenience, instead of the condition in (3.1) we consider the Farey dissection of the continuum

$$
\mathbb{R}=\bigcup_{a / q} \mathcal{A}_{a / q} \text { with } \mathcal{A}_{a / q}=\left[\frac{a+a^{-}}{q+q^{-}}, \frac{a+a^{+}}{q+q^{+}}\right),
$$

where $a^{-} / q^{-}<a / q<a^{+} / q^{+}$are consecutive fractions in the Farey sequence of a fixed order, extended periodically. In our case we take the order to be $\left\lfloor N^{1 / 2}\right\rfloor$. In this way we can assign to each $x$ a unique $a_{x} / q_{x}$ such that

$$
x \in \mathcal{A}_{a_{x} / q_{x}} \text { with } q_{x} \leq N^{1 / 2} .
$$

Proposition 3.1. Let $Q$ be an integral positive definite binary quadratic form and $\alpha, \beta$ arbitrary real numbers. Then for every $N \geq 2$ and $x \in \mathbb{R}$ satisfying (3.2), we have

$$
\sum_{0 \leq n \leq N} r_{\alpha, \beta}(n) e(n x) \ll \frac{N(\log N)^{2}}{q_{x}+N\left|q_{x} x-a_{x}\right|},
$$

where

$$
r_{\alpha, \beta}(n)=\sum_{Q\left(n_{1}, n_{2}\right)=n} e\left(\alpha n_{1}+\beta n_{2}\right),
$$

uniformly in $\alpha$ and $\beta$.

In what follows we introduce and estimate some auxiliary functions that will be used in the proof of Proposition 3.1. To simplify the notation all the bounds will be expressed in terms of the 1-periodic function

$$
B(t)=\min \left(N,\|t\|^{-1}\right),
$$

where in this section $\|\cdot\|$ means the distance to the nearest integer. 
Lemma 3.2. For $N \in \mathbb{Z}^{+}$

$$
\sum_{0 \leq n \leq N} e(n t) e^{2 \pi n / N} \ll B(t) .
$$

Proof: The left hand side is a geometric series which can be readily bounded.

Let $A$ be the matrix of the integral quadratic form $Q$ and consider

$$
\theta_{\vec{v}}(z)=\sum_{n \geq 0} r_{\alpha, \beta}(n) e(n z) \quad \text { with } \vec{v}=\frac{1}{2} A^{-1}\left(\begin{array}{l}
\alpha \\
\beta
\end{array}\right) .
$$

This holomorphic function in the upper half plane corresponds to a Jacobi modular form for some special values of $\alpha$ and $\beta$. The reason to parametrize it in terms of $\vec{v}$ is to make the transformation formula, which we state next, as concise as possible. The proof is adapted from $[\mathbf{2 7}$, Chapter 4], where it is presented in the more general context of indefinite forms.

Lemma 3.3. If $z$ and $w$, in the upper half plane, are related by a modular transformation

$$
w=\frac{a z+b}{c z+d} \quad \text { with } \gamma:=\left(\begin{array}{ll}
a & b \\
c & d
\end{array}\right) \in \mathrm{SL}_{2}(\mathbb{Z}) \text { and } c \neq 0
$$

then

$$
j_{\gamma}(z) \theta_{\vec{v}}(z)=\frac{\delta(\gamma, \vec{v})}{2 \sqrt{\operatorname{det} A}} \sum_{\vec{l} \in \mathcal{L}} G_{\vec{l}} \sum_{\vec{x} \in \mathbb{Z}^{2}+\vec{l}} e(w Q(\vec{x}+c \vec{v})-2 a \vec{x} \cdot A \vec{v}),
$$

where $\delta$ is a certain function with $|\delta|=1, j_{\gamma}(z)=c z+d$, as usual, and $G_{\vec{l}}$ is a normalized Gauss sum associated to each representative of the quotient of lattices $\mathcal{L}=\frac{1}{2} A^{-1} \mathbb{Z}^{2} / \mathbb{Z}^{2}$, namely

$$
G_{\vec{l}}=\frac{1}{c} \sum_{\vec{g}(\bmod c)} e\left(-\frac{a Q(\vec{l}+d \vec{g})}{c}\right) .
$$

Proof: By the definition of $\theta_{\vec{v}}(z)$ and separating the classes modulo $c$,

$\theta_{\vec{v}}(z)=\sum_{\vec{n} \in \mathbb{Z}^{2}} e(z Q(\vec{n})+2 \vec{n} \cdot A \vec{v})=\sum_{\vec{g}(\bmod c)} \sum_{\vec{m} \in \mathbb{Z}^{2}} e(z Q(c \vec{m}+\vec{g})+2(c \vec{m}+\vec{g}) \cdot A \vec{v})$.

Writing $\left(j_{\gamma}(z)-d\right) / c$ instead of $z$ and completing squares, the phase can be expressed as $P_{1}+P_{2}$ with

$P_{1}=\frac{j_{\gamma}(z)}{c} Q\left(c \vec{m}+\vec{g}+\frac{c \vec{v}}{j_{\gamma}(z)}\right) \quad$ and $\quad P_{2}=-\frac{c}{j_{\gamma}(z)} Q(\vec{v})-\frac{d}{c} Q(c \vec{m}+\vec{g})$. 
Note that $P_{2}$ does not change modulo 1 when $\vec{m}$ varies and we can put $\vec{m}=\overrightarrow{0}$. On the other hand, by Proposition 10.1 of $[\mathbf{2 0}]$,

$$
\sum_{\vec{m} \in \mathbb{Z}^{2}} e\left(P_{1}\right)=\frac{i(\operatorname{det} A)^{-1 / 2}}{2 c j_{\gamma}(z)} \sum_{\vec{m} \in \mathbb{Z}^{2}} e\left(-\frac{Q\left(A^{-1} \vec{m} / 2\right)}{c j_{\gamma}(z)}+c^{-1}\left(\vec{g}+\frac{c \vec{v}}{j_{\gamma}(z)}\right) \cdot \vec{m}\right) .
$$

Under the change of variables $\vec{x}=\frac{1}{2} A^{-1}(-\vec{m})$ with $\vec{x}=\vec{n}+\vec{l}$, where $\vec{l} \in \mathcal{L}$ and $\vec{n} \in \mathbb{Z}^{2}$, this phase corresponds to

$$
P_{3}=-\frac{Q(\vec{x})+2 c \vec{v} \cdot A \vec{x}}{c j_{\gamma}(z)}-\frac{2}{c} \vec{g} \cdot A \vec{x} .
$$

Then, substituting $\left(j_{\gamma}(z)\right)^{-1}=-c w+a$ in $P_{2}$ and $P_{3}$,

$$
\begin{aligned}
e\left(P_{2}+P_{3}\right)=e(w Q(\vec{x})+2(c w & -a) \vec{v} \cdot A \vec{x}+c(c w-a) Q(\vec{v})) \\
& \times e\left(-\frac{a}{c} Q(\vec{x})-\frac{2}{c} \vec{x} \cdot A \vec{g}-\frac{d}{c} Q(\vec{g})\right) .
\end{aligned}
$$

The last exponential is $e(-a Q(\vec{x}+d \vec{g}) / c)$ because $a d \equiv 1(\bmod c)$, and when we sum on $\vec{g}$ we obtain $c G_{\vec{l}}$. It only remains to note that the argument of the first exponential can be written as $w Q(\vec{x}+c \vec{v})-2 a \vec{v}$. $A \vec{x}-\operatorname{ac} Q(\vec{v})$.

Lemma 3.4. With the previous notation and $x$ as in (3.2),

$$
\theta_{\vec{v}}(x+i / N) \ll q_{x}^{-1} B\left(x-a_{x} / q_{x}\right),
$$

the implicit constant depending solely on the form $Q$.

Proof: Let $\gamma \in \mathrm{SL}_{2}(\mathbb{Z})$ be a matrix with lower row $(q,-p)$, i.e. $\gamma(p / q)=$ $\infty$, and apply Lemma 3.3. Since $\Im w=\Im z /\left|j_{\gamma}(z)\right|^{2}$ with $\left|j_{\gamma}(z)\right|=|q z-p|$ and $G_{\vec{l}} \ll 1[\mathbf{2 1}$, Lemma 20.12], the right hand side of the transformation formula can be estimated term-wise, obtaining the bound

$$
\theta_{\vec{v}}(z) \ll|q z-p|^{-1} \sum_{x \in \mathbb{Z}^{2}+\mathcal{L}} e^{-K Q(\vec{x}+q \vec{v})}
$$

under the hypothesis $\Im z \geq K|q z-p|^{2}$. Note the sumset $\mathbb{Z}^{2}+\mathcal{L}=\{\vec{n}+\vec{l}$ : $\left.\vec{n} \in \mathbb{Z}^{2}, \vec{l} \in \mathcal{L}\right\}$ depends only on $Q$, and since the sum can be seen as a continuous double periodic function evaluated at $q \vec{v}$ it must be uniformly bounded. We have therefore proved $\theta_{\vec{v}}(z) \ll|q z-p|^{-1}$ uniformly in $\vec{v}$ when $|q z-p|^{2}=O(\Im z)$. Choosing now $z=x+i / N$ and $p / q=a_{x} / q_{x}$ it is enough to note that $|t+i / N|^{-1} \ll B(t)$ for $|t| \leq 1 / 2$.

Lemma 3.5. For $t \in \mathbb{R}$ we have

$$
(B * B)(t):=\int_{-1 / 2}^{1 / 2} B(u) B(t-u) d u \ll N \frac{\log (2+N\|t\|)}{2+N\|t\|} .
$$


Proof: Cauchy's inequality gives $(B * B)(t) \ll \int_{0}^{1}|B|^{2} \ll N$. Using this and the symmetry, we can assume $2 N^{-1} \leq t \leq 1 / 2$. If $0<u<1 / 2$, then the distance from $t$ to $u$ is always smaller than or equal to the distance from $t$ to $-u$. Hence $B(t-u) \geq B(t+u)$ and $(B * B)(t) \leq$ $2 \int_{0}^{1 / 2} B(u) B(t-u) d u$. This last integral is bounded above by

$\int_{0}^{N^{-1}} \frac{N d u}{t-u}+\int_{N^{-1}}^{t-N^{-1}} \frac{d u}{u(t-u)}+\int_{t-N^{-1}}^{t+N^{-1}} \frac{N d u}{u}+\int_{t+N^{-1}}^{1 / 2+N^{-1}} \frac{d u}{u(u-t)}$, that gives $O\left(t^{-1} \log (N t)\right)$ evaluating or estimating the integrals.

Proof of Proposition 3.1: Assume for convenience $-1 / 2 \leq x<1 / 2$ and let $D_{N}^{*}(t)$ be the left hand side in (3.3). We have

$$
\sum_{0 \leq n \leq N} r_{\alpha, \beta}(n) e(n x)=\int_{-1 / 2}^{1 / 2} \sum_{n \geq 0} r_{\alpha, \beta}(n) e(n(y+i / N)) D_{N}^{*}(x-y) d y .
$$

By Lemma 3.2 and Lemma 3.4

$$
\sum_{0 \leq n \leq N} r_{\alpha, \beta}(n) e(n x) \ll \sum_{a / q} q^{-1} \int_{\mathcal{A}_{a / q}} B(y-a / q) B(x-y) d y,
$$

where $\cup \mathcal{A}_{a / q}$ is the Farey dissection of $[-1 / 2,1 / 2]$ of order $\left\lfloor N^{1 / 2}\right\rfloor$ as before. Trivially

$$
\mathcal{I}_{a / q}:=\int_{\mathcal{A}_{a / q}} B(y-a / q) B(x-y) d y \leq(B * B)(x-a / q) .
$$

If $a / q=a_{x} / q_{x}$ we employ Lemma 3.5 (with an extra logarithm to absorb an error term appearing later) to get

$$
\mathcal{I}_{a_{x} / q_{x}} \ll \frac{N(\log N)^{2}}{1+N\left|x-a_{x} / q_{x}\right|} .
$$

In the rest of the cases $|x-a / q| \gg N^{-1}$ is assured and $\mathcal{I}_{a / q} \ll \mid x-$ $a /\left.q\right|^{-1} \log N$ by Lemma 3.5. Substituting in (3.4)

$$
\sum_{0 \leq n \leq N} r_{\alpha, \beta}(n) e(n x) \ll \frac{N(\log N)^{2}}{q_{x}+N\left|q_{x} x-a_{x}\right|}+(\log N) \sum_{a / q \neq a_{x} / q_{x}}|q x-a|^{-1} .
$$

Each summand attains its maximum when $x$ is one of the end-points of $\mathcal{A}_{a_{x} / q_{x}}$, both of which are rational numbers $A / Q$ with $Q \asymp N^{1 / 2}$. Hence doubling the sum, it suffices to bound

$$
\sum_{a / q \neq a_{x} / q_{x}}|q A / Q-a|^{-1}=Q \sum_{m \leq N} m^{-1} \#\{a / q: A q-Q a= \pm m\} .
$$

The last cardinality is $O(1)$ and introducing this bound in (3.5), the result follows. 


\section{Paraboloids}

We are ready to prove the first statement of Theorem 1.1 in the following stronger form:

Theorem 4.1. Let $\mathcal{P}$ be as in (1.3) with $d \geq 3$. Assume that the coefficient matrix $A=\left(a_{i j}\right)$ of $Q$ satisfies $a_{12} / a_{11}, a_{22} / a_{11} \in \mathbb{Q}$. Then for each fixed $\epsilon>0$,

$$
\mathcal{N}(R)=|\mathcal{P}| R^{d}+O\left(R^{d-2+\epsilon}\right)
$$

holds uniformly for $0<c \ll 1<R$ and $\vec{\beta} \in \mathbb{R}^{d-1}$.

The proof is divided in two steps: first we deal with the threedimensional case where we can exploit the full rationality of $Q$, and then we extend the result to higher dimensions. The uniformity in $c$ and $\vec{\beta}$ is crucial for the second step to succeed, as these parameters have to be taken depending on $R$.

Proof of Theorem 4.1, case $d=3$ : Rescaling $R$ and $c$ we may suppose that $Q$ is integral. We may also assume that the vector $\left(\alpha_{1}, \alpha_{2}\right)=R \vec{\beta}$ lies in $[0,1) \times[0,1)$, since $\mathcal{N}(R)$ is 1 -periodic in these variables. Finally we assume $c>4 R^{-2}$ because $\mathcal{N}(R)-|\mathcal{P}| R^{3}=O(R)$ when $c \ll R^{-1}$. This last claim is straightforward, since $|\mathcal{P}| \ll c^{2}$, while $\mathcal{N}(R)$ is counting points with integer coordinates in at most $2 c R+1$ circles of radius proportional to $c^{1 / 2} R$.

We have

$$
\begin{aligned}
\frac{1}{2} \mathcal{N}(R) & =\sum_{n_{1}, n_{2}} \sum^{\prime}\left(\left\lfloor f\left(n_{1}, n_{2}\right)\right\rfloor+\frac{1}{2}\right) \\
& =\sum_{n_{1}, n_{2}} \sum^{\prime} f\left(n_{1}, n_{2}\right)-\sum_{n_{1}, n_{2}} \sum^{\prime} \psi\left(f\left(n_{1}, n_{2}\right)\right),
\end{aligned}
$$

where $f(x, y)=\left(c R^{2}-Q\left(x+\alpha_{1}, y+\alpha_{2}\right)\right) / R, \psi(x)=x-\lfloor x\rfloor-1 / 2$, and the prime indicates that the double summation is restricted to $Q\left(n_{1}+\right.$ $\left.\alpha_{1}, n_{2}+\alpha_{2}\right) \leq c R^{2}$.

Let $\chi$ be the characteristic function of $Q\left(x+\alpha_{1}, y+\alpha_{2}\right) \leq c R^{2}$. Applying Euler-Maclaurin formula firstly in $n_{2}$ and secondly in $n_{1}$, we have

$$
\begin{aligned}
\sum_{n_{1}, n_{2}} \sum^{\prime} f\left(n_{1}, n_{2}\right) & =\sum_{\left|n_{1}\right| \ll R \sqrt{c}}\left(\int \chi\left(n_{1}, y\right) f\left(n_{1}, y\right) d y+O(1)\right) \\
& =\int \chi(x, y) f(x, y) d y d x+O(R)
\end{aligned}
$$

and the last integral is, of course, $\frac{1}{2}|\mathcal{P}| R^{3}$. 
It is well known (see for instance Vaaler's lemma in $[\mathbf{2 5}, \S 1.2]$ ) that for any $M \in \mathbb{Z}^{+}$there exist trigonometric polynomials $Q^{ \pm}(x)=$ $\sum_{|m| \leq M} a_{m}^{ \pm} e(m x)$ such that $Q^{-}(x) \leq \psi(x) \leq Q^{+}(x)$ with $a_{0}^{ \pm} \ll M^{-1}$ and $a_{m}^{ \pm} \ll m^{-1}$. Taking $M=\left\lfloor c^{1 / 2} R\right\rfloor$ we get from (4.1)

$$
\mathcal{N}(R)=|\mathcal{P}| R^{3}+O(\mathcal{E}(R))+O\left(R^{1+\epsilon}\right)
$$

with

$$
\mathcal{E}(R)=\sum_{m \leq M} \frac{1}{m}\left|\sum_{n_{1}, n_{2}} \sum^{\prime \prime} e\left(\frac{m}{R} Q\left(n_{1}+\alpha_{1}, n_{2}+\alpha_{2}\right)\right)\right| .
$$

The double prime indicates we have replaced the summation domain to $Q\left(n_{1}, n_{2}\right) \leq M^{2}$, at the cost of adding and removing at most $O(M)$ terms. Subdividing into dyadic intervals, there exists $K \leq M$ such that

$$
\mathcal{E}(R) \ll K^{-1} M^{\epsilon} \sum_{K \leq m<2 K}\left|\sum_{n_{1}, n_{2}} \sum^{\prime \prime} e\left(\frac{m}{R} Q\left(n_{1}+\alpha_{1}, n_{2}+\alpha_{2}\right)\right)\right| .
$$

By Proposition 3.1,

$$
\mathcal{E}(R) \ll K^{-1} M^{2+\epsilon} \sum_{K \leq m<2 K}\left(q+M^{2}|q m / R-a|\right)^{-1},
$$

where $a=a(m)$ and $q=q(m)$ are determined by (3.2) with $x=m / R$. In particular, we have

$$
|m q-a R| \leq \frac{R}{M} .
$$

If $K>R / M$ then $0 \neq a \ll R$ and for each fixed $a$ we have that $m$ divides an integer in an interval of length $O(R / M)$. This leaves $O\left(R^{1+\epsilon} / M\right)$ possibilities for $m$. Neglecting the term $M^{2}|q m / R-a|$ in (4.3) and using $q \asymp R a / K$,

$$
\mathcal{E}(R) \ll K^{-1} M^{2+\epsilon} \sum_{a \ll R} R^{1+\epsilon} M^{-1}(R a / K)^{-1} \ll R^{1+\epsilon} .
$$

If $K \leq R / M$ the argument of divisibility fails when $a / q=0 / 1$. These terms can be estimated directly in (4.3), while the previous argument can be applied to those with $a \neq 0$, yielding again $\mathcal{E}(R) \ll R^{1+\epsilon}$.

Proof of Theorem 4.1, case $d>3$ : Write $\vec{x}=\left(\vec{x}_{1}, \vec{x}_{2}\right)$ and $\vec{\beta}=\left(\vec{\beta}_{1}, \vec{\beta}_{2}\right)$ with $\vec{x}_{1}, \vec{\beta}_{1} \in \mathbb{R}^{2}$ and $\vec{x}_{2}, \vec{\beta}_{2} \in \mathbb{R}^{d-3}$. Completing squares,

$$
Q(\vec{x}+\vec{\beta})=Q_{1}\left(\vec{x}_{1}+\vec{\gamma}\right)+Q_{2}\left(\vec{x}_{2}+\vec{\beta}_{2}\right),
$$

where $\vec{\gamma}$ depends linearly on $\left(\vec{x}_{2}, \vec{\beta}_{1}, \vec{\beta}_{2}\right)$ and the matrix of $Q_{1}$ is $\left(a_{i j}\right)_{i, j=1}^{2}$. 
Given $\vec{n}_{2} \in \mathbb{Z}^{d-3}$, let us denote by $\mathcal{P}_{\vec{n}_{2}}$ the three-dimensional slice of $\mathcal{P}$ obtained by fixing $\vec{x}_{2}=\vec{n}_{2} / R$, and by $\mathcal{N}_{\vec{n}_{2}}(R)$ the number of lattice points it contains after being dilated with scale factor $R$. By the three-dimensional case of this theorem and the decomposition (4.4),

$$
\mathcal{N}(R)=\sum_{\vec{n}_{2}} \mathcal{N}_{\vec{n}_{2}}(R)=\sum_{\vec{n}_{2}}\left|\mathcal{P}_{\vec{n}_{2}}\right| R^{3}+O\left(R^{d-2+\epsilon}\right),
$$

both sums extended to the domain $Q_{2}\left(\vec{n}_{2}+R \vec{\beta}_{2}\right) \leq c R^{2}$. A simple computation shows

$$
\left|\mathcal{P}_{\vec{n}_{2}}\right|=\frac{\pi}{\sqrt{D}}\left(c-Q_{2}\left(\vec{n}_{2} / R+\vec{\beta}_{2}\right)\right)^{2},
$$

where $D$ is the determinant of (the Hessian matrix of) $Q_{1}$. Applying the Euler-Maclaurin formula iteratively in one variable at a time we find

$$
\frac{\pi}{\sqrt{D}} \sum_{\vec{n}_{2}}\left(c-Q_{2}\left(\vec{n}_{2} / R+\vec{\beta}_{2}\right)\right)^{2}=\frac{\pi}{\sqrt{D}} \int\left(c-Q_{2}\left(\vec{x}_{2} / R\right)\right)^{2} d \vec{x}_{2}+O\left(R^{d-5}\right)
$$

and the main term in the right hand side is $|\mathcal{P}| R^{d-3}$.

\section{Some $\Omega$-results}

Let us start considering first the two-dimensional parabolic region $\mathcal{P}_{2}$ introduced in $\S 2$. The simple estimate $\mathcal{N}_{2}(R)-\left|\mathcal{P}_{2}\right| R^{2}=\Omega\left(R^{1 / 2}\right)$, already contained in $[\mathbf{2 6}]$, follows by noting that for some values of $R$ there are at least $\Omega\left(R^{1 / 2}\right)$ points lying on the boundary of $\mathcal{P}_{2}$ dilated by $R$. Indeed, given any positive integer $M$, let $R=M^{2}$ and consider the points $\left(k M, \pm\left(M^{2}-k^{2}\right)\right)$ with $-M \leq k \leq M$. The following more precise one-sided $\Omega$-results are a consequence of Corollaries 2.2 and 2.3.

Proposition 5.1. The error term $\mathcal{E}(R)=\mathcal{N}_{2}(R)-\left|\mathcal{P}_{2}\right| R^{2}$ satisfies

$$
\mathcal{E}(R)=\Omega_{+}\left(R^{1 / 2}\right) \quad \text { and } \quad \mathcal{E}(R)=\Omega_{-}\left(R^{1 / 2} \log \log R\right) .
$$

Proof: The first statement follows by taking $N$ a square in Corollary 2.2. For the second one we remark that the main result of $[\mathbf{1}]$ asserts that there are infinitely many primes $p \equiv 3(\bmod 4)$ satisfying $h(-p) / \sqrt{p} \gg \log \log p$. It suffices to take $N=p$ for any such prime $p$ in Corollary 2.3.

The upper bound $h(-d) / \sqrt{d} \ll \log \log d$ is known to hold under the generalized Riemann hypothesis [24]. Any hope to obtain a better $\Omega_{-}$-result from Corollary 2.3 therefore must take advantage of the sum of class numbers, and for this we need uniform lower bounds over certain families of discriminants. Fortunately Heath-Brown proved an astonishing result that, in some way, shows the absence of exceptional 
zeros for large multiples of some primes in a fixed set [15]. Even more astonishing is the short and elementary proof of this fact. In its original form (see [4] for an enhaced version with the same proof) the result claims that if $S$ is a fixed set of more than $505^{2}$ odd primes then for any sufficiently large integer $d$ there exists a prime $p_{d} \in S$ satisfying $L\left(1, \chi_{-p_{d} d}\right) \gg(\log d)^{-1 / 9}$. Using this we prove the following one-sided $\Omega$-result:

Proposition 5.2. We have

$\mathcal{N}_{2}(R)-\left|\mathcal{P}_{2}\right| R^{2}=\Omega_{-}\left(R^{1 / 2} \exp (c \sqrt{\log R} / \log \log R)\right) \quad$ for any $c<\sqrt{2}$.

Proof: Let $S$ be the set of the first $505^{2}+1$ primes $p \equiv 3(\bmod 4)$ and fix an integer $d_{0}$ large enough so that the aforementioned result of Heath-Brown holds for any $d \geq d_{0}$. Choose $N=N^{\prime} \prod_{p \in S} p$ in Corollary 2.3, where $N^{\prime}$ is the product of the primes $p \equiv 1(\bmod 4)$ in the interval $\left[d_{0}, x\right]$ for any large $x$. Then by the class number formula and $[\mathbf{1 5}]$,

$$
\sum_{\substack{d \mid N \\ d \equiv 3(4)}} \omega_{d} h(-d) \gg \sum_{\substack{d \mid N^{\prime} \\ d \neq 1}} \frac{\sqrt{p_{d} d}}{(\log d)^{1 / 9}} \gg \frac{\sqrt{N^{\prime}}}{(\log N)^{1 / 9}} \prod_{p \mid N^{\prime}}\left(1+p^{-1 / 2}\right) .
$$

The result now follows by noting that the logarithm of the product over the primes is asymptotically $\sqrt{2 \log N^{\prime}} / \log \log N^{\prime}$ and $N^{\prime} \gg N$.

We now prove some $\Omega$-results for higher dimensional centered rational paraboloids i.e., those of the form

(5.1) $\mathcal{P}=\left\{(\vec{x}, y) \in \mathbb{R}^{d-1} \times \mathbb{R}:|y| \leq c-Q(\vec{x})\right\} \quad$ with $c \in \mathbb{Q}$ and $Q$ rational.

Theorem 5.3. The lattice point discrepancy $\mathcal{N}(R)-|\mathcal{P}| R^{d}$ for $\mathcal{P}$ as in (5.1) is $\Omega\left(R^{d-2} \eta(R)\right)$, where

$$
\eta(R)= \begin{cases}\exp \left(K \frac{\log R}{\log \log R}\right) & \text { for any } K<\log 2 \text { when } d=3, \\ \log \log R & \text { when } d=4, \\ \sqrt{\log \log R} & \text { when } d=5 \\ 1 & \text { when } d \geq 6 .\end{cases}
$$

This proves that our main result is sharp in the sense that the $\epsilon$ in the exponent cannot be dropped in the low dimensional cases. Note that when $d \geq 6$ and $\mathcal{P}$ is as in (5.1) the lattice point discrepancy is actually $O\left(R^{d-2}\right)$, as shown by applying Euler-Maclaurin summation to the corresponding asymptotics for the number of lattice points in the dilated $(d-1)$-dimensional ellipsoid $\{Q(\vec{x}) \leq 1\}$ (see, for instance, $[\mathbf{1 0}$, Satz 1 of $\S 21])$. For general paraboloids, however, our method does not provide an answer as to whether the $\epsilon$ is really necessary for $d \geq 6$. 
To deduce Theorem 5.3 we consider $B(R)$ with $R \in \mathbb{Z}^{+}$, the number of lattice points on the boundary of $\mathcal{P}$ dilated by $R$. Clearly, an $\Omega$-result for $B(R)$ readily implies the same $\Omega$-result for the lattice point discrepancy. We will therefore prove Theorem 5.3 in the stronger form $B(R)=\Omega\left(R^{d-2} \eta(R)\right)$. Some reductions first: note that without loss of generality we may assume $c \in \mathbb{Z}$, and let $Q=\frac{a}{b} Q^{*}$ where $Q^{*}$ is a primitive integral quadratic form. For each $\vec{n} \in \mathbb{Z}^{d-2}$ with $Q^{*}(\vec{n})=R n$ and $a b n \leq c R$ we have that the lattice point $(b \vec{n}, c R-a b n)$ is counted by $B(R)$. In other words,

$$
B(R) \geq \sum_{n \leq \alpha R} r_{Q^{*}}(R n) \quad \text { with } \alpha=\frac{c}{a b},
$$

where $r_{Q^{*}}(k)$ is the number of representations of $k$ by the quadratic form $Q^{*}$. For the remaining proofs we will not need to refer to $Q$ anymore, and therefore we will write $Q$ instead of $Q^{*}$ for the sake of notational simplicity.

Proof of Theorem 5.3, case $d=3$ : Let $r_{1}, r_{2}, \ldots, r_{k}$ be the solutions of

$$
Q(r, 1) \equiv 0 \quad(\bmod R)
$$

and for each $1 \leq j \leq k$ and a fixed $0<\delta<1 / 2$ define

$$
C_{j}=\left\{(x, y) \in \mathbb{Z}^{2}:|y| \leq \delta R,|x| \leq \delta R, x \equiv r_{j} y \quad(\bmod R)\right\} .
$$

Choosing $\delta^{2}<\frac{1}{2} \lambda^{-1} \alpha$ with $\lambda$ the greatest eigenvalue of the matrix of $Q$, we have that $Q$ maps $C_{j}$ into multiples of $R$ less than $\alpha R^{2}$. Hence the sum in (5.2) is at least $\# \cup_{j} C_{j}$. If we restrict $y$ to $\operatorname{gcd}(y, R)=1$ then the sets $C_{j}$ become disjoint, consequently

$$
B(R) \geq k \min _{j} \# C_{j}-k \#\{y \in \mathbb{Z}:|y|<R, \operatorname{gcd}(y, R)>1\} .
$$

For each fixed $j$, consider the remainders of $0 r_{j}, 1 r_{j}, 2 r_{j}, \ldots,\lfloor\delta R\rfloor r_{j}$ when divided by $R$. By the pigeonhole principle, if we subdivide $[0, R)$ into $\left\lceil\delta^{-1}\right\rceil$ equal subintervals, at least $\delta R /\left\lceil\delta^{-1}\right\rceil$ of the remainders lie in the same subinterval. In this way, we have at least $\delta R /\left\lceil\delta^{-1}\right\rceil$ pairs $\left(u_{\ell}, v_{\ell}\right)$ such that $0 \leq v_{\ell} \leq \delta R$ and all $u_{\ell} \equiv r_{j} v_{\ell}$ lie in the same subinterval of length $R /\left\lceil\delta^{-1}\right\rceil$. Hence $\left(u_{\ell}-u_{1}, v_{\ell}-v_{1}\right) \in C_{j}$ and it follows $\# C_{j} \geq$ $\delta R /\left\lceil\delta^{-1}\right\rceil$. In this way, (5.3) assures

$$
B(R) \geq k \frac{\delta^{2} R}{1+\delta}+2 k(\varphi(R)-R)
$$

where $\varphi$ stands for Euler's totient function. 
For large $x$, take $R$ as the product of the primes $x \leq p \leq 2 x$ such that $\left(\frac{\Delta}{p}\right)=1$ where $\Delta$ is the discriminant of $Q$. Again by the prime number theorem in arithmetic progressions, we have

$$
\log R \sim \frac{x}{2} \quad \text { and } \quad \frac{\varphi(R)}{R}=\prod_{p \mid R}\left(1-p^{-1}\right)=1+O\left(\frac{1}{\log x}\right) .
$$

The congruence $Q(r, 1) \equiv 0$ admits two solutions modulo each of these primes $p$. Then by our choice of $R$ we have that $k$ equals 2 to the number of such primes that is at least $(\log R) / \log (2 x)$. Substituting this and (5.5) in (5.4), we get the expected result.

Proof of Theorem 5.3, case $d=4$ : Combining Theorem 1 of [4] and Theorem 2 of $[8]$ we have

$$
r_{Q}(n)=r_{Q}^{\mathrm{gen}}(n)+O\left(n^{13 / 28+\epsilon}\right) \text { for } n \notin \mathcal{S},
$$

where $\mathcal{S}$ is a finite union of sets of the form $\left\{t_{j} m^{2}: m \in \mathbb{Z}\right\}$ for some $t_{j} \in \mathbb{Z}$. Here $r_{Q}^{\text {gen }}$ is the average number of representations by forms belonging to the same genus as $Q$ that can be computed with Siegel mass formula (see $[\mathbf{2 1}, \S 20.4]$ for the definitions and details). In Lemma 6 of [6] this formula was written as

$$
r_{Q}^{\mathrm{gen}}(n)=\frac{4 \pi \sqrt{2 n}}{\sqrt{D}} \sum_{d^{2} \mid n} d^{-1} U\left(n / d^{2}\right) L\left(1, \chi_{-2 D n / d^{2}}\right),
$$

where $D$ is the determinant of $Q, L$ is the $L$-function corresponding to the Kronecker symbol $\chi_{m}$ modulo $m=-2 D n / d^{2}$, and $U$ is a certain $8 D^{2}$-periodic function which is nonnegative and not identically zero.

Assume $\operatorname{gcd}(R, 2 D)=1$ and for each $d^{2} \mid R$ choose $n_{d}$ such that $U\left(n_{d} R / d^{2}\right) \neq 0$, then (5.6) and (5.7) together with (5.8) imply

$$
B(R) \gg R \sum_{d^{2} \mid R} d^{-1} \mathcal{L}_{d}(R)+O\left(R^{27 / 14+\epsilon}\right)
$$

where

$$
\mathcal{L}_{d}(R)=\sum_{n \in \mathcal{A}} L\left(1, \chi_{-2 D R n / d^{2}}\right)
$$

with

$$
\mathcal{A}=\left\{n \asymp R: R n \notin \mathcal{S}, n \equiv n_{d} \quad\left(\bmod 8 D^{2}\right)\right\} .
$$

If $\mathcal{L}_{d}(R) \gg R$, choosing $R=\prod_{2 D<p \leq x} p^{2}$ we have $\log R \sim 2 x$ and

$$
B(R) \gg R^{2} \prod_{2 D<p \leq x}\left(1+p^{-1}\right)+O\left(R^{27 / 14+\epsilon}\right) \gg R^{2} \log \log R .
$$


It remains to prove $\mathcal{L}_{d}(R) \gg R$. Expanding the $L$-functions, we can write $\mathcal{L}_{d}(R)$ as

$$
\begin{aligned}
S_{1}+S_{2}+S_{3}:= & \sum_{m_{1}} \frac{1}{m_{1}} \sum_{n \in \mathcal{A}} \chi_{d_{n}}\left(m_{1}\right) \\
& +\sum_{m_{2}} \frac{\chi-2 D R^{\prime}\left(m_{2}\right)}{m_{2}} \sum_{n \in \mathcal{A}} \chi_{n}\left(m_{2}\right)+\sum_{n \in \mathcal{A}} \sum_{m_{3}} \frac{\chi_{d_{n}}\left(m_{3}\right)}{m_{3}},
\end{aligned}
$$

where $d_{n}=-2 D R^{\prime} n, R^{\prime}=R / d^{2}, m_{1}$ runs over the squares in $\left[1, R^{1+\epsilon}\right]$, $m_{2}$ over the non-squares coprime to $2 D R^{\prime}$ in the same interval, and $m_{3}>R^{1+\epsilon}$. Trivially, $S_{1} \gg R$. By Pólya-Vinogradov inequality $S_{3} \ll \sum_{n \in \mathcal{A}} R^{-\epsilon} \ll R^{1-\epsilon}$. There are $O\left(R^{1 / 2}\right)$ values of $n \asymp R$ with $R n \in$ $\mathcal{S}$ that when added to $\mathcal{A}$ give a negligible contribution $O\left(R^{1 / 2} \log R\right)$ to $S_{2}$, and hence we can drop the condition $R n \notin \mathcal{S}$ in $S_{2}$. On the other hand, the congruence condition $n \equiv n_{d}$ can be detected inserting $\sum_{\chi} \chi(n) \bar{\chi}\left(n_{d}\right) / \varphi\left(8 D^{2}\right)$ where $\chi$ runs over the characters modulo $8 D^{2}$. Since $\operatorname{gcd}\left(m_{2}, 2 D R^{\prime}\right)=1, \psi(n)=\chi(n) \chi_{n}\left(m_{2}\right)$ is a nonprincipal character modulo $8 D^{2} m_{2}$ and Pólya-Vinogradov inequality proves $S_{2} \ll$ $R^{1 / 2+\epsilon}$. Therefore $\mathcal{L}_{d}(R) \sim S_{1} \gg R$.

Proof of Theorem 5.3, case $d \geq 5$ : For $d \geq 6$ we have by Corollary 11.3 of [20] the estimate $r_{Q}(m) \asymp m^{(d-3) / 2}$ as long as $m$ is sufficiently large and $Q(\vec{x}) \equiv m$ is solvable modulo $2^{7} D^{3}$ with $D$ the determinant of $Q$. Taking $m=R n$ with $R$ a large multiple of $2^{7} D^{3}$, both conditions are fulfilled and the result follows from (5.2).

If $d=5$, Corollary 11.3 of [20] gives for $2^{7} D^{3} \mid R$

$$
B(R) \gg R \sum_{n \leq \alpha R} n \prod_{p \mid R n}\left(1+\chi_{D}(p) p^{-1}\right) \quad \text { with } \chi_{D}(p)=\left(\frac{D}{p}\right) .
$$

Let $P_{D}$ the product of the primes $p \leq x$ such that $\chi_{D}(p)=1$. By the prime number theorem in arithmetic progressions, we have

$$
\log P_{D} \sim \frac{x}{2} \text { and } \prod_{p \mid P_{D}}\left(1+p^{-1}\right) \gg \sqrt{\log x} \sim \sqrt{\log \log P_{D}} .
$$

Choosing $R=2^{7} D^{3} P_{D}$ in (5.9), we have

$$
B(R) \gg R \prod_{p \mid P_{D}}\left(1+p^{-1}\right) \cdot \sum_{n \leq \alpha R} n \prod_{p \mid n}\left(1-p^{-1}\right) .
$$

The sum equals that of $\varphi(n)$ that is comparable to $R^{2}$.

Acknowledgement. We are grateful to A. D. Martínez for his useful comments on an early version of this article. 


\section{References}

[1] P. T. Bateman, S. Chowla, and P. Erdös, Remarks on the size of $L(1, \chi)$, Publ. Math. Debrecen 1 (1950), 165-182.

[2] V. Bentkus and F. Götze, On the lattice point problem for ellipsoids, Acta Arith. 80(2) (1997), 101-125. DOI : 10.4064/aa-80-2-101-125.

[3] V. Bentkus and F. Götze, Lattice point problems and distribution of values of quadratic forms, Ann. of Math. (2) 150(3) (1999), 977-1027. DOI : 10.2307/ 121060.

[4] V. Blomer, Uniform bounds for Fourier coefficients of theta-series with arithmetic applications, Acta Arith. 114(1) (2004), 1-21. DOI: 10.4064/aa114-1-1.

[5] J. Bourgain and N. Watt, Mean square of zeta function, circle problem and divisor problem revisited, Preprint (2017). arXiv: 1709.04340.

[6] F. Chamizo, E. Cristóbal, and A. Ubis, Lattice points in rational ellipsoids, $J$. Math. Anal. Appl. 350(1) (2009), 283-289. DOI: 10.1016/j.jmaa.2008.09.051.

[7] F. Chamizo and H. Iwaniec, On the Gauss mean-value formula for class number, Nagoya Math. J. 151 (1998), 199-208. DOI : 10.1017/S0027763000025241.

[8] W. Duke And R. Schulze-Pillot, Representation of integers by positive ternary quadratic forms and equidistribution of lattice points on ellipsoids, Invent. Math. 99(1) (1990), 49-57. DOI : 10.1007/BF01234411.

[9] H. Fiedler, W. Jurkat, And O. Körner, Asymptotic expansions of finite theta series, Acta Arith. 32(2) (1977), 129-146. DOI : 10.4064/aa-32-2-129-146.

[10] F. Fricker, "Einführung in die Gitterpunktlehre", Lehrbücher und Monographien aus dem Gebiete der Exakten Wissenschaften (LMW) 73, Birkhäuser Verlag, Basel-Boston, Mass., 1982. DOI : 10.1007/978-3-0348-7185-3.

[11] F. Götze, Lattice point problems and values of quadratic forms, Invent. Math. 157(1) (2004), 195-226. DOI : 10.1007/s00222-004-0366-3.

[12] J. Guo, On lattice points in large convex bodies, Acta Arith. 151(1) (2012), 83-108. DOI : $10.4064 /$ aa151-1-6.

[13] J. L. HAFNER, New omega theorems for two classical lattice point problems, Invent. Math. 63(2) (1981), 181-186. DOI : 10.1007/BF01393875.

[14] G. H. Hardy and J. E. Littlewood, Some problems of diophantine approximation. I. The fractional part of $n^{k} \theta$, Acta Math. 37(1) (1914), 155-191. DOI: 10.1007/BF02401833; II. The trigonometrical series associated with the elliptic $\vartheta$-functions, Acta Math. 37(1) (1914), 193-239. DOI : 10.1007/BF02401834.

[15] D. R. Heath-Brown, Ternary quadratic forms and sums of three square-full numbers, in: "Séminaire de Théorie des Nombres, Paris 1986-87", Progr. Math. 75, Birkhäuser Boston, Boston, MA, 1988, pp. 137-163.

[16] D. R. Heath-Brown, Lattice points in the sphere, in: "Number Theory in Progress", Vol. 2 (Zakopane-Kościelisko, 1997), de Gruyter, Berlin, 1999, pp. 883-892. DOI : 10.1515/9783110285581.883.

[17] K. Henriot and K. Hughes, On restriction estimates for discrete quadratic surfaces, Preprint (2016). arXiv:1611.00720.

[18] M. N. Huxley, Exponential sums and lattice points III, Proc. London Math. Soc. (3) 87(3) (2003), 591-609. DOI : 10.1112/S0024611503014485.

[19] A. Ivić, E. Krätzel, M. Kühleitner, and W. G. NowaK, Lattice points in large regions and related arithmetic functions: recent developments in a very classic topic, in: "Elementare und Analytische Zahlentheorie", Schr. Wiss. Ges. 
Johann Wolfgang Goethe Univ. Frankfurt am Main 20, Franz Steiner Verlag Stuttgart, Stuttgart, 2006, pp. 89-128.

[20] H. Iwaniec, "Topics in Classical Automorphic Forms", Graduate Studies in Mathematics 17, American Mathematical Society, Providence, RI, 1997. DOI: $10.1090 / \mathrm{gsm} / 017$.

[21] H. Iwaniec and E. Kowalski, "Analytic Number Theory", American Mathematical Society Colloquium Publications 53, American Mathematical Society, Providence, RI, 2004. DOI : 10.1090/coll/053.

[22] E. Krätzel, Lattice points in elliptic paraboloids, J. Reine Angew. Math. 1991(416) (1991), 25-48. DOI : 10.1515/crll.1991.416.25.

[23] E. KRÄTZEL, Weighted lattice points in three-dimensional convex bodies and the number of lattice points in parts of elliptic paraboloids, J. Reine Angew. Math. 1997(485) (1997), 11-23. DOI : 10.1515/crll.1997.485.11.

[24] J. E. Littlewood, On the class-number of the corpus $P(\sqrt{ }-k)$, Proc. London Math. Soc. (2) 27(1) (1928), 358-372. DOI: 10.1112/plms/s2-27.1.358.

[25] H. L. Montgomery, "Ten Lectures on the Interface between Analytic Number Theory and Harmonic Analysis", CBMS Regional Conference Series in Mathematics 84, Published for the Conference Board of the Mathematical Sciences, Washington, DC; by the American Mathematical Society, Providence, RI, 1994. DOI : $10.1090 / \mathrm{cbms} / 084$.

[26] V. N. Popov, The number of integer points under a parabola, (Russian), Mat. Zametki 18(5) (1975), 699-704.

[27] C. L. Siegel, "Lectures on Quadratic Forms", Notes by K. G. Ramanathan, Tata Institute of Fundamental Research Lectures on Mathematics 7, Tata Institute of Fundamental Research, Bombay, 1967.

[28] K. Soundararajan, Omega results for the divisor and circle problems, Int. Math. Res. Not. 2003(36) (2003), 1987-1998. DOI : 10.1155/S1073792803130309.

Fernando Chamizo

Departamento de Matemáticas, Universidad Autónoma de Madrid, 28049 Madrid, Spain

E-mail address: fernando.chamizo@uam.es

Carlos Pastor

Instituto de Ciencias Matemáticas, Nicolás Cabrera 13-15, 28049 Madrid, Spain

E-mail address: carlos.pastor@icmat.es

Primera versió rebuda el 4 de setembre de 2017, darrera versió rebuda el 30 de gener de 2018. 